\title{
Distribution, depletion and recovery of docosahexaenoic acid are region-specific in rat brain
}

\author{
Ying Xiao ${ }^{1,2}$, Yu Huang ${ }^{3}$ and Zhen-Yu Chen ${ }^{1 *}$ \\ ${ }^{1}$ Food and Nutritional Sciences Programme, Department of Biochemistry, The Chinese University of Hong Kong, Shatin, NT, \\ Hong Kong, China \\ ${ }^{2}$ Department of Nutrition and Food Hygiene, University Health Science Center, Peking University, Beijing, China \\ ${ }^{3}$ Department of Physiology, The Chinese University of Hong Kong, Shatin, NT, Hong Kong, China
}

(Received 21 February 2005 - Revised 31 May 2005 - Accepted 7 June 2005)

\begin{abstract}
The present study examined: (i) age-induced regional changes in fatty acid composition of brain phospholipids; (ii) $\alpha$-linolenic acid deficiency-induced regional depletion and recovery of DHA in the brain. DHA and arachidonic acid (AA) did not distribute evenly in the brain. In weaning and adult rats, the region with the highest DHA percentage was the cortex whereas the medulla had the lowest DHA percentage. In the aged rats, both the cortex and cerebellum were the regions with the highest DHA percentage whereas in the neonatal rats, the striatum had the greatest percentage of DHA, and the hypothalamus and hippocampus had the least percentage of DHA. Regarding AA, the hippocampus was the region that had the highest percentage whereas the medulla was the region with the lowest percentage except for the neonatal rats, whose cerebellum, hypothalamus, striatum and midbrain had AA percentage lower than hippocampus and cortex. DHA was not proportionally depleted in various regions of brain when the rats were maintained on an $n$-3-deficient diet for two generations. The results demonstrated that the cortex, hippocampus, striatum, cerebellum and hypothalamus had DHA depleted by $>71 \%$, whereas the midbrain and medulla had only 64 and $57 \%$ DHA depleted, respectively. The most important observation was that the diet reversal for 12 weeks resulted in complete DHA recovery in all regions except for the medulla where the recovery was only $62 \%$. It was concluded that the location of DHA, $n-3$ deficiency-induced DHA depletion and reversibility of DHA deficiency across the brain were region-specific.
\end{abstract}

Arachidonic acid: Brain: Cerebellum: Cortex: Docosahexaenoic acid

DHA (22:6n-3) and arachidonic acid (AA; $20: 4 n-6)$ are important for the structural development of the mammalian central nervous system and are accumulated in large amounts in the developing brain and retina. DHA and AA can be synthesised from their respective short-chain precursors, $\alpha$-linolenic acid (LNA; $18: 3 n-3)$ and linoleic acid $(18: 2 n-6)$, via the desaturase and elongase pathway. LNA and linoleic acid are regarded as essential fatty acids, since mammals cannot synthesise them and have to obtain them from the diet. However, LNA and linoleic acid are quantitatively minor in any mammalian brain (Sastry, 1985). DHA and AA contents in the growing brain are largely determined by dietary fatty acid composition. Depletion of DHA can be induced by a diet deficient in LNA. Fortunately, the deficiency is not permanent and is reversible by re-supplementation of LNA or preformed DHA in the diet (Connor et al. 1990; Moriguchi et al. 2001).

Previous research has shown that the addition of DHA or DHA and AA combined into infant formula are associated with an improved performance in visual acuity, visual recognition memory, learning ability, Bayley mental developmental index, and developmental quotient scores of infants (Uauy et al. 1990; Carlson et al. 1993; Agostoni et al. 1995; Carlson \& Werkman, 1996; Willatts et al. 1998; Birch et al. 2000; Innis, 2004). However, the beneficial effect associated with supplementation of DHA and AA on neural functions of infants has not always been consistently observed in other trials (Innis et al. 1994; Auestad et al. 1997; Jorgensen et al. 1998; Makrides et al. 1995; Lucas et al. 1999, 2002). An association between DHA deficiency and functional deficits in the nervous system has also been demonstrated in animal studies. DHA deficiency was typically induced by feeding a diet low in $n-3$ fatty acids during development. DHA depletion in the brain led rodents to show poor performance in the Morris water maze, shock avoidance, spatial task performance, olfactory discrimination, and exploratory behaviour (Bourre et al. 1989; Enslen et al. 1991; Greiner et al. 1999; Moriguchi et al. 2000). DHA deficiency induced by a low-LNA diet also caused abnormal electroretinograms in rats, cats and rhesus monkeys (Wheeler et al. 1975; Neuringer et al. 1986; Pawlosky et al. 1997). However, other trials found no difference in performance for spatial tasks between the control and DHA-depleted mice (Wainwright et al. 1994, 1997).

Fatty acid composition in various species is well characterised but regional distribution of DHA and AA in the brain has not been thoroughly examined (Carrie et al. 2000). The present study was to: (i) examine differences in fatty acid composition of sub-brain regions including the cerebellum, medulla, hypothalamus, striatum, hippocampus, cortex and midbrain of rats; (ii) investigate whether LNA deficiency-induced DHA depletion was region-specific in these sub-regions; (iii) most importantly, exam- 
ine the regional difference in DHA recovery in the brain after diet reversal from an $n$-3-deficient diet to an $n$-3-adequate diet.

\section{Materials and methods}

\section{Age and regional distribution of fatty acids in rat brain}

Female Sprague-Dawley rats (Laboratory Experimental Animal Service, The Chinese University of Hong Kong, Shatin, Hong Kong) were housed (two rats per cage) in an animal room at $23^{\circ} \mathrm{C}$ with $12-12 \mathrm{~h}$ light-dark cycles. The rats consumed a rodent chow diet (PMI Nutrition International, LLC, Brentwood, MO, USA) that contained $60 \mathrm{~g}$ fat $/ \mathrm{kg}$. The fatty acid composition of the chow diet is shown in Table 1. The rats were killed under $\mathrm{CO}_{2}$ anaesthesia at day $3(8.6(\mathrm{SD} 0.3) \mathrm{g})$, day $21(55.0$ (SD 2.6) g), week 10 (231.6 (SD 19.0) g) and month 18 (412.5 (SD 29.9) g). The whole brain was retained and then dissected into cerebellum, medulla, hypothalamus, striatum, hippocampus, cortex and midbrain according to the procedure previously described by Glowinski \& Iversen (1966).

\section{n-3 Deficiency-induced regional depletion of docosahexaenoic acid from rat brain}

DHA deficiency was induced by feeding an $n$-3-deficient diet for two generations. In brief, the experiment started with weaning SpragueDawley female rats. They were divided into two groups (ten per group) and placed on either an $n-3$ fatty acid-adequate ( $n-3$ Adq) diet or an $n$ - 3 fatty acid-deficient ( $n-3$ Def) diet. The rats were allowed free access to diet and tap water. When the rats were 10 weeks old (n-3 Adq, 286.2(SD 19.4) g; $n$-3 Def, 272.2(SD 23.1)g), they (F1 generation) were mated with Sprague-Dawley male rats (12 weeks). After weaning, the pups (F2 generation) were placed on their corresponding maternal diet. At week 5 , the F2 female rats (n-3 Adq, 110.0 (SD 26.2) g; n-3 Def, 111.2 (SD 13.4) g) were killed under $\mathrm{CO}_{2}$ anaesthesia. The brain was removed and dissected into cerebellum, medulla, hypothalamus, striatum, hippocampus, cortex and midbrain for the fatty acid analysis.

\section{Reversibility of docosahexaenoic acid deficiency}

The F2 n-3 Def female rats at week 5 were switched to the $n-3$ Adq diet for 12 weeks whereas the F2 n-3 Adq female rats were maintained on the $n-3$ Adq diet for the same period of

Table 1. Fatty acid composition (\%) of rodent chow diet (Data averaged from three determinations)

\begin{tabular}{lrr}
\hline Fatty acids & Mean & SD \\
\hline $16: 0$ & $18 \cdot 9$ & $0 \cdot 1$ \\
$18: 0$ & $7 \cdot 0$ & $0 \cdot 1$ \\
$16: 1 n-7$ & $1 \cdot 8$ & $0 \cdot 1$ \\
$18: 1 n-9$ & $27 \cdot 5$ & $1 \cdot 0$ \\
$20: 1 n-9$ & $0 \cdot 7$ & $0 \cdot 1$ \\
$18: 2 n-6$ & $35 \cdot 0$ & $0 \cdot 1$ \\
$20: 2 n-6$ & $0 \cdot 2$ & 0.0 \\
$20: 3 n-6$ & $0 \cdot 2$ & 0.0 \\
$20: 4 n-6$ & $<0 \cdot 1$ & 0.0 \\
$18: 3 n-3$ & $3 \cdot 1$ & $0 \cdot 1$ \\
$20: 5 n-3$ & $0 \cdot 8$ & 0.0 \\
$22: 5 n-3$ & $0 \cdot 1$ & 0.0 \\
$22: 6 n-3$ & 0.6 & 0.0 \\
\hline
\end{tabular}

time. Five rats from each group were killed at every 3 weeks and sub-regions were dissected for fatty acid analysis.

\section{Diets}

The composition of the $n-3$ Adq diet and $n-3$ Def diet was similar except for fat (Table 2). All diet ingredients were purchased from Harlan Teklad (Madison, WI, USA) except for the maize starch, oils and sucrose, which were obtained from a local supermarket. Both diets contained $60 \mathrm{~g}$ fat $/ \mathrm{kg}$. The $n-3$ Adq diet contained (per kg diet): $42.3 \mathrm{~g}$ coconut oil, $8.4 \mathrm{~g}$ rapeseed oil, $6.8 \mathrm{~g}$ sunflower-seed oil and $2.5 \mathrm{~g}$ flaxseed oil. The $n-3$ Def diet contained only $44.2 \mathrm{~g}$ coconut oil and $15.8 \mathrm{~g}$ sunflower-seed oil (Table 2 ). The fatty acid composition of the two diets was similar except for LNA $(18: 3 n-3)$, which accounted for $3.49 \%$ in the $n-3$ Adq diet but was less than $0.04 \%$ of total fatty acids in the $n-3$ Def diet (Table 3 ).

\section{Fatty acid analysis}

Total lipids of brain regions were extracted using chloroformmethanol $(2: 1, \mathrm{v} / \mathrm{v})$ containing $2 \%$ butyrated hydroxytoluene (Sigma Chemical Co., St Louis, MO, USA) as an antioxidant. L-Phosphatidylcholine diheptadecanoyl (Sigma Chemical Co.) was added as an internal standard to quantify total phospholipids (PL). Neutral lipid TLC $(20 \times 20 \mathrm{~cm}$ plates pre-coated with $250 \mu \mathrm{m}$ silica gel 60A; Macherey-Nagel Gmbh \& Co. KG, Düren, Germany) was applied to separate total PL by using a developing solvent system of hexane-diethyl ether-acetic acid $(80: 20: 1$, by vol.). Total PL were recovered from the TLC plate, and their fatty acids were converted to the corresponding methyl esters using $14 \%$ boron trifluoride in methanol (Sigma Chemical Co.) under $\mathrm{N}_{2}$ gas at $90^{\circ} \mathrm{C}$ for $60 \mathrm{~min}$.

The fatty acid methyl esters were analysed on a flexible silica capillary column (Innowax 19091N-213, $30 \mathrm{~m} \times 0.32 \mathrm{~mm}$ internal diameter; J\&W Scientific, Folsom, CA, USA) in an HP 5980 series II gas-liquid chromatograph equipped with a flame-ionisation detector (Hewlett Packard, Palo Alto, CA, USA). Column temperature was programmed from 180 to $230^{\circ} \mathrm{C}$ at a rate of $2^{\circ} \mathrm{C} / \mathrm{min}$ and then held for $5 \mathrm{~min}$. Injector and detector temperature were set at 250 and $300^{\circ} \mathrm{C}$, respectively. The carrier gas used was $\mathrm{He}$ at a head pressure of $100 \mathrm{kPa}$. Identification of each fatty acid methyl ester was made by comparison of retention time of authentic standards (Sigma Chemical Co.).

Table 2. Composition ( $\mathrm{g} / \mathrm{kg}$ diet) of the $n-3$ adequate diet and $n$-3-deficient diet

\begin{tabular}{|c|c|c|}
\hline & $n$-3-Adequate diet & $n$-3-Deficient diet \\
\hline Maize starch & 571.0 & 571.0 \\
\hline Casein & $235 \cdot 0$ & $235 \cdot 0$ \\
\hline Sucrose & $50 \cdot 0$ & $50 \cdot 0$ \\
\hline Mineral mix $(\mathrm{AIN}-76)^{\star}$ & $35 \cdot 0$ & $35 \cdot 0$ \\
\hline Cellulose & $32 \cdot 0$ & $32 \cdot 0$ \\
\hline Vitamin mix (AIN-76A)* & $10 \cdot 0$ & $10 \cdot 0$ \\
\hline Choline bitartrate & $4 \cdot 0$ & $4 \cdot 0$ \\
\hline DL-Methionine & $3 \cdot 0$ & 3.0 \\
\hline Total fat & $60 \cdot 0$ & $60 \cdot 0$ \\
\hline Coconut oil & $42 \cdot 3$ & $44 \cdot 2$ \\
\hline Rapeseed oil & 8.4 & 0 \\
\hline Sunflower-seed oil & $6 \cdot 8$ & $15 \cdot 8$ \\
\hline Flaxseed oil & 2.5 & 0 \\
\hline
\end{tabular}

* Supplied by Harlan Teklad, Madison, WI, USA. 
Table 3. Fatty acid composition (\% of total fatty acids) of the $n$-3 adequate diet and $n$-3-deficient diet

\begin{tabular}{lcc}
\hline Fatty acid & $n$-3-Adequate diet & $n$-3-Deficient diet \\
\hline $12: 0$ & $33 \cdot 3$ & $34 \cdot 6$ \\
$14: 0$ & $13 \cdot 5$ & $14 \cdot 6$ \\
$16: 0$ & $9 \cdot 8$ & $8 \cdot 7$ \\
$18: 0$ & $3 \cdot 5$ & $3 \cdot 2$ \\
$18: 1 n-9$ & $15 \cdot 0$ & $17 \cdot 1$ \\
$18: 2 n-6$ & $17 \cdot 5$ & $17 \cdot 7$ \\
$18: 3 n-3$ & $3 \cdot 5$ & $<0 \cdot 1$ \\
Others & $3 \cdot 9$ & $4 \cdot 1$ \\
\hline
\end{tabular}

\section{Statistics}

Data are expressed as means and standard deviations. Where applicable, one-way ANOVA was used followed by using Fisher's least significant difference method to statistically evaluate significant differences in fatty acid composition among the various regions in the brain using SPSS 10.0 (SPSS Inc., Chicago, IL, USA). Differences were considered significant when $P<0.05$.

\section{Results \\ Regional distribution of phospholipid fatty acids}

The regional distribution of fatty acids in PL of sub-brain regions of neonatal rats (day 3 ) is shown in Table 4. It appeared that the striatum had the greatest percentage of DHA (12.6) while the hypothalamus and hippocampus had the least percentage of DHA (10.7 and $10 \cdot 8$, respectively). AA $(20: 4 n-6)$ in all the regions was similar, but statistically in the hippocampus and cortex it was greater than that in the cerebellum, hypothalamus, striatum and midbrain.

A regional difference in DHA distribution was observed in weaning rats aged $21 \mathrm{~d}$ (Table 4). The cortex had the greatest

Table 4. Major fatty acids (\% of total) of phospholipids of sub-regions including the cerebellum, medulla, hypothalamus, striatum, hippocampus, cortex and midbrain of rats aged $3 \mathrm{~d}, 21 \mathrm{~d}, 10$ weeks and 18 months $(n 10)$

(Mean values and standard deviations for ten rats)

\begin{tabular}{|c|c|c|c|c|c|c|c|c|c|c|c|c|c|c|}
\hline & \multicolumn{2}{|c|}{ Cerebellum } & \multicolumn{2}{|c|}{ Medulla } & \multicolumn{2}{|c|}{ Hypothalamus } & \multicolumn{2}{|c|}{ Striatum } & \multicolumn{2}{|c|}{ Hippocampus } & \multicolumn{2}{|c|}{ Cortex } & \multicolumn{2}{|c|}{ Midbrain } \\
\hline & Mean & $\mathrm{SD}$ & Mean & $\mathrm{SD}$ & Mean & $\mathrm{SD}$ & Mean & $\mathrm{SD}$ & Mean & $\mathrm{SD}$ & Mean & $\mathrm{SD}$ & Mean & SD \\
\hline \multicolumn{15}{|l|}{$16: 0$} \\
\hline $3 d$ & $32 \cdot 4^{\mathrm{A}, \mathrm{C}}$ & 0.5 & $31 \cdot 6^{A, d}$ & 0.8 & $32 \cdot 4^{\mathrm{A}, \mathrm{b}, \mathrm{c}}$ & 0.5 & $33 \cdot 2^{\mathrm{A}, \mathrm{a}}$ & 0.1 & $33.0^{\mathrm{A}, \mathrm{a}, \mathrm{b}}$ & 0.4 & $32 \cdot 9^{\mathrm{A}, \mathrm{a}, \mathrm{b}}$ & 0.2 & $33 \cdot 3^{\mathrm{A}, \mathrm{a}}$ & 0.4 \\
\hline $21 d$ & $20 \cdot 5^{B, d}$ & 0.6 & $17 \cdot 2^{\mathrm{B}, \mathrm{f}}$ & 0.7 & $24 \cdot 5^{\mathrm{B}, \mathrm{c}}$ & 0.7 & $23 \cdot 4^{\mathrm{B}, \mathrm{d}}$ & 0.5 & $25 \cdot 2^{\mathrm{B}, \mathrm{b}}$ & 0.5 & $26 \cdot 0^{\mathrm{B}, \mathrm{a}}$ & 0.6 & $21 \cdot 9^{\mathrm{B}, \mathrm{e}}$ & 0.8 \\
\hline 10 weeks & $20 \cdot 2^{B, b, c}$ & 0.3 & $14.5^{\mathrm{C}, \mathrm{e}}$ & 0.3 & $21 \cdot 0^{\mathrm{C}, \mathrm{b}}$ & 0.9 & $19 \cdot 5^{\mathrm{C}, \mathrm{c}}$ & 0.9 & $21 \cdot 6^{\mathrm{C}, \mathrm{a}, \mathrm{b}}$ & 0.5 & $22 \cdot 4^{\mathrm{C}, \mathrm{a}, \mathrm{b}}$ & 0.5 & $17 \cdot 9^{\mathrm{C}, \mathrm{d}}$ & 0.1 \\
\hline 18 months & $19 \cdot 2^{\mathrm{C}, \mathrm{c}}$ & 0.9 & $13 \cdot 9^{\mathrm{C}, \mathrm{e}}$ & 0.4 & $19 \cdot 1^{\mathrm{D}, \mathrm{C}}$ & 0.9 & $18 \cdot 6^{\mathrm{C}, \mathrm{c}}$ & $1 \cdot 2$ & $20 \cdot 3^{C, b}$ & 0.5 & $21 \cdot 8^{\mathrm{C}, \mathrm{a}}$ & 0.4 & $17 \cdot 3^{\mathrm{C}, \mathrm{d}}$ & 0.2 \\
\hline \multicolumn{15}{|l|}{$18: 0$} \\
\hline $3 d$ & $16 \cdot 8^{\mathrm{D}, \mathrm{c}}$ & 0.2 & $17 \cdot 4^{\mathrm{B}, \mathrm{b}}$ & 0.5 & $18 \cdot 0^{\mathrm{B}, \mathrm{a}}$ & 0.5 & $17 \cdot 4^{\mathrm{B}, \mathrm{b}, \mathrm{c}}$ & 0.2 & $17 \cdot 6^{\mathrm{B}, \mathrm{a}, \mathrm{b}}$ & 0.4 & $17 \cdot 1^{\mathrm{B}, \mathrm{C}}$ & 0.3 & $17 \cdot 1^{\mathrm{C}, \mathrm{c}}$ & 0.3 \\
\hline $21 d$ & $20 \cdot 3^{A, d}$ & 0.3 & $20 \cdot 5^{A, c}$ & 0.3 & $21 \cdot 9^{\mathrm{A}, \mathrm{a}}$ & 0.2 & $21 \cdot 8^{\mathrm{A}, \mathrm{a}}$ & 0.5 & $21 \cdot 4^{\mathrm{A}, \mathrm{b}}$ & 0.3 & $21 \cdot 5^{A, b}$ & 0.4 & $21 \cdot 6^{\mathrm{A}, \mathrm{a}, \mathrm{b}}$ & 0.3 \\
\hline 10 weeks & $19 \cdot 8^{\mathrm{B}, \mathrm{d}}$ & 0.2 & $17 \cdot 7^{\mathrm{B}, \mathrm{e}}$ & 0.3 & $21 \cdot 3^{A, b, c}$ & 0.1 & $22 \cdot 1^{\mathrm{A}, \mathrm{a}}$ & 0.2 & $21.9^{A, a, b}$ & 0.6 & $21 \cdot 5^{A, b}$ & 0.7 & $20 \cdot 7^{\mathrm{B}, \mathrm{c}}$ & 0.2 \\
\hline 18 months & $18 \cdot 7^{\mathrm{C}, \mathrm{d}}$ & 0.1 & $17 \cdot 3^{\mathrm{B}, \mathrm{e}}$ & 0.1 & $21 \cdot 4^{\mathrm{A}, \mathrm{b}}$ & 0.6 & $21 \cdot 0^{A, b, c}$ & 0.2 & $21 \cdot 9^{\mathrm{A}, \mathrm{a}}$ & 0.2 & $21 \cdot 6^{\mathrm{A}, \mathrm{a}, \mathrm{b}}$ & 0.1 & $20 \cdot 1^{\mathrm{B}, \mathrm{C}}$ & 0.1 \\
\hline \multicolumn{15}{|l|}{$18: 1 n-9$} \\
\hline $3 d$ & $13 \cdot 2^{\mathrm{C}, \mathrm{a}}$ & 0.5 & $13 \cdot 2^{\mathrm{C}, \mathrm{a}}$ & 0.4 & $12 \cdot 9^{\mathrm{C}, \mathrm{a}}$ & 0.3 & $11 \cdot 2^{\mathrm{C}, \mathrm{c}}$ & 0.1 & $12 \cdot 0^{\mathrm{C}, \mathrm{b}}$ & 0.4 & $11 \cdot 3^{\mathrm{D}, \mathrm{c}}$ & 0.2 & $11 \cdot 9^{\mathrm{D}, \mathrm{b}}$ & 0.3 \\
\hline $21 d$ & $18 \cdot 0^{\mathrm{B}, \mathrm{b}}$ & 0.5 & $22 \cdot 3^{\mathrm{B}, \mathrm{a}}$ & 0.7 & $15 \cdot 7^{\mathrm{B}, \mathrm{C}}$ & 0.5 & $15 \cdot 9^{\mathrm{B}, \mathrm{C}}$ & 0.4 & $14 \cdot 6^{\mathrm{B}, \mathrm{d}}$ & 0.5 & $13 \cdot 1^{\mathrm{C}, \mathrm{e}}$ & 0.6 & $17 \cdot 9^{\mathrm{C,b}}$ & 1.0 \\
\hline 10 weeks & $20 \cdot 7^{A, b}$ & 0.5 & $26 \cdot 5^{\mathrm{A}, \mathrm{a}}$ & 0.4 & $20 \cdot 4^{\mathrm{A}, \mathrm{C}}$ & 0.9 & $20 \cdot 2^{A, C}$ & 1.4 & $18 \cdot 7^{\mathrm{A}, \mathrm{d}}$ & 0.7 & $17 \cdot 8^{\mathrm{B}, \mathrm{d}}$ & 0.9 & $23 \cdot 9^{\mathrm{B}, \mathrm{b}}$ & 0.5 \\
\hline \multicolumn{15}{|l|}{$18: 2 n-6$} \\
\hline $3 d$ & $1 \cdot 3^{\mathrm{A}, \mathrm{a}}$ & 0.2 & $1 \cdot 1^{A, b, c}$ & 0.1 & $0.9^{\mathrm{A}, \mathrm{C}}$ & 0.1 & $0.9^{\mathrm{A}, \mathrm{C}}$ & 0.1 & $1 \cdot 1^{A, b, c}$ & 0.1 & $1 \cdot 2^{\mathrm{A}, \mathrm{b}}$ & 0.1 & $1 \cdot 0^{\mathrm{A}, \mathrm{C}}$ & 0.1 \\
\hline $21 d$ & $1 \cdot 4^{\mathrm{A}, \mathrm{a}}$ & 0.1 & $1 \cdot 3^{A, b}$ & 0.1 & $0.9^{\mathrm{A}, \mathrm{e}}$ & 0.2 & $1.0^{\mathrm{A}, \mathrm{d}}$ & 0.1 & $0.9^{\mathrm{B}, \mathrm{e}}$ & 0.1 & $1 \cdot 0^{A, C}$ & 0.1 & $0.9^{A, d}$ & 0.1 \\
\hline 10 weeks & $1 \cdot 0^{\mathrm{B}, \mathrm{a}}$ & 0.1 & $0 \cdot 6^{\mathrm{B}, \mathrm{b}}$ & 0.0 & $0.5^{\mathrm{B}, \mathrm{b}, \mathrm{c}}$ & 0.0 & $0 \cdot 6^{\mathrm{B}, \mathrm{b}}$ & 0.0 & $0.5^{\mathrm{C}, \mathrm{c}}$ & 0.1 & $0.6^{\mathrm{B}, \mathrm{b}, \mathrm{c}}$ & 0.2 & $0 \cdot 6^{\mathrm{B}, \mathrm{b}}$ & 0.0 \\
\hline 18 months & $0.9^{\mathrm{B}, \mathrm{a}}$ & 0.1 & $0 \cdot 6^{\mathrm{B}, \mathrm{b}}$ & 0.1 & $0 \cdot 5^{\mathrm{B}, \mathrm{c}}$ & 0.1 & $0 \cdot 6^{\mathrm{B}, \mathrm{C}}$ & 0.0 & $0 \cdot 5^{\mathrm{C}, \mathrm{c}}$ & 0.1 & $0 \cdot 7^{\mathrm{B}, \mathrm{b}}$ & 0.0 & $0 \cdot 7^{\mathrm{B}, \mathrm{b}, \mathrm{c}}$ & 0.0 \\
\hline \multicolumn{15}{|l|}{$20: 4 n-6$} \\
\hline $3 d$ & $12 \cdot 4^{\mathrm{A}, \mathrm{b}}$ & 0.4 & $12 \cdot 8^{\mathrm{A}, \mathrm{a}, \mathrm{b}}$ & 0.6 & $12 \cdot 3^{A, b}$ & 0.5 & $12 \cdot 3^{\mathrm{A}, \mathrm{b}}$ & 0.2 & $12 \cdot 9^{\mathrm{A}, \mathrm{a}}$ & 0.2 & $12 \cdot 9^{\mathrm{A}, \mathrm{a}}$ & 0.2 & $12 \cdot 4^{\mathrm{A}, \mathrm{b}}$ & 0.4 \\
\hline $21 d$ & $9 \cdot 2^{\mathrm{B}, \mathrm{e}}$ & 0.2 & $8 \cdot 0^{\mathrm{B}, \mathrm{f}}$ & 0.3 & $11 \cdot 4^{\mathrm{B}, \mathrm{C}}$ & 0.3 & $12 \cdot 3^{\mathrm{A}, \mathrm{b}}$ & 0.2 & $13 \cdot 0^{\mathrm{A}, \mathrm{a}}$ & 0.3 & $12 \cdot 8^{\mathrm{A}, \mathrm{a}}$ & 0.5 & $10 \cdot 2^{\mathrm{B}, \mathrm{d}}$ & 0.7 \\
\hline 10 weeks & $7 \cdot 2^{C, d}$ & 0.2 & $5 \cdot 6^{\mathrm{C}, \mathrm{e}}$ & 0.2 & $9 \cdot 2^{C, c}$ & 0.7 & $10 \cdot 3^{\mathrm{B}, \mathrm{b}}$ & 0.3 & $11 \cdot 4^{\mathrm{B}, \mathrm{a}}$ & 0.4 & $10 \cdot 0^{\mathrm{B}, \mathrm{b}}$ & 0.4 & $7 \cdot 6^{C, b}$ & 0.2 \\
\hline 18 months & $6 \cdot 1^{\mathrm{D}, \mathrm{e}}$ & 0.2 & $5 \cdot 0^{\mathrm{D}, \mathrm{f}}$ & 0.2 & $7 \cdot 9^{\mathrm{D}, \mathrm{c}}$ & 0.2 & $9 \cdot 0^{\mathrm{C}, \mathrm{b}}$ & 0.2 & $10 \cdot 4^{\mathrm{C}, \mathrm{a}}$ & 0.3 & $9 \cdot 1^{\mathrm{C}, \mathrm{b}}$ & 0.3 & $6 \cdot 8^{\mathrm{D}, \mathrm{d}}$ & 0.0 \\
\hline \multicolumn{15}{|l|}{$22: 4 n-6$} \\
\hline $3 d$ & $3 \cdot 1^{A, b}$ & 0.2 & $3 \cdot 3^{\mathrm{A}, \mathrm{a}}$ & $0 \cdot 2$ & $3 \cdot 5^{\mathrm{B}, \mathrm{a}}$ & 0.3 & $2 \cdot 6^{\mathrm{B}, \mathrm{c}, \mathrm{a}}$ & 0.2 & $2 \cdot 8^{\mathrm{C}, \mathrm{C}}$ & 0.2 & $2 \cdot 7^{\mathrm{B}, \mathrm{c}, \mathrm{a}}$ & 0.2 & $2 \cdot 9^{\mathrm{B}, \mathrm{b}, \mathrm{c}}$ & 0.2 \\
\hline $21 d$ & $3 \cdot 2^{A, c, d}$ & 0.9 & $3 \cdot 1^{A, d}$ & 0.1 & $4 \cdot 4^{\mathrm{A}, \mathrm{a}}$ & 0.2 & $3 \cdot 1^{A, d}$ & 0.1 & $3 \cdot 4^{\mathrm{A}, \mathrm{C}}$ & 0.1 & $3 \cdot 3^{A, c, d}$ & 0.1 & $3 \cdot 6^{A, b}$ & 0.1 \\
\hline 10 weeks & $2 \cdot 4^{\mathrm{B}, \mathrm{f}}$ & 0.1 & $2 \cdot 7^{\mathrm{B}, \mathrm{e}}$ & 0.1 & $4 \cdot 3^{\mathrm{A}, \mathrm{a}}$ & 0.1 & $3 \cdot 3^{A, b, c}$ & 0.1 & $3 \cdot 5^{\mathrm{A}, \mathrm{b}}$ & 0.1 & $3 \cdot 0^{A, d}$ & 0.1 & $3 \cdot 2^{A, C}$ & 0.1 \\
\hline 18 months & $1 \cdot 7^{\mathrm{C}, \mathrm{f}}$ & 0.1 & $1.9^{\mathrm{C}, \mathrm{e}}$ & 0.1 & $3 \cdot 7^{\mathrm{C}, \mathrm{a}}$ & 0.1 & $2 \cdot 8^{\mathrm{C}, \mathrm{C}}$ & 0.1 & $3 \cdot 2^{\mathrm{A}, \mathrm{b}}$ & 0.1 & $2 \cdot 5^{\mathrm{B}, \mathrm{d}}$ & 0.3 & $2 \cdot 5^{\mathrm{B}, \mathrm{d}}$ & 0.1 \\
\hline \multicolumn{15}{|l|}{$22: 5 n-6$} \\
\hline $3 d$ & $1.4^{\mathrm{A}, \mathrm{b}}$ & 0.2 & $1.5^{\mathrm{A}, \mathrm{a}, \mathrm{b}}$ & 0.2 & $1 \cdot 8^{\mathrm{A}, \mathrm{a}}$ & 0.2 & $1 \cdot 8^{\mathrm{A}, \mathrm{a}}$ & 0.2 & $1 \cdot 7^{\mathrm{A}, \mathrm{a}}$ & 0.2 & $1 \cdot 8^{\mathrm{A}, \mathrm{a}}$ & 0.2 & $1 \cdot 7^{\mathrm{A}, \mathrm{b}, \mathrm{c}}$ & 0.2 \\
\hline $21 d$ & $0 \cdot 6^{\mathrm{B}, \mathrm{C}}$ & 0.1 & $0 \cdot 3^{\mathrm{B}, \mathrm{d}}$ & 0.1 & $0.8^{\mathrm{B}, \mathrm{a}}$ & 0.1 & $0.6^{\mathrm{B}, \mathrm{C}}$ & 0.1 & $0 \cdot 7^{\mathrm{B}, \mathrm{b}}$ & 0.1 & $0 \cdot 7^{\mathrm{B}, \mathrm{b}}$ & 0.1 & $0 \cdot 6^{\mathrm{B}, \mathrm{c}}$ & 0.2 \\
\hline \multicolumn{15}{|l|}{$22: 6 n-3$} \\
\hline $3 d$ & $10 \cdot 9^{C, b, c}$ & 0.6 & $11 \cdot 6^{\mathrm{B}, \mathrm{b}}$ & 0.8 & $10 \cdot 7^{\mathrm{B}, \mathrm{C}}$ & 0.8 & $12 \cdot 6^{\mathrm{C}, \mathrm{a}}$ & 0.4 & $10 \cdot 8^{\mathrm{B}, \mathrm{c}}$ & 0.4 & $11 \cdot 4^{\mathrm{B}, \mathrm{b}, \mathrm{c}}$ & 0.5 & $11 \cdot 5^{\mathrm{B}, \mathrm{b}}$ & 0.7 \\
\hline $21 d$ & $13 \cdot 8^{\mathrm{B}, \mathrm{b}}$ & 0.4 & $11 \cdot 7^{\mathrm{B}, \mathrm{d}}$ & 0.3 & $13 \cdot 1^{A, C}$ & 0.6 & $13 \cdot 5^{\mathrm{B}, \mathrm{b}, \mathrm{c}}$ & 0.3 & $13 \cdot 9^{A, b}$ & 0.7 & $15 \cdot 3^{\mathrm{A}, \mathrm{a}}$ & 0.6 & $13 \cdot 6^{A, b}$ & 0.5 \\
\hline 10 weeks & $14 \cdot 2^{\mathrm{B}, \mathrm{b}}$ & 0.4 & $10 \cdot 7^{\mathrm{C}, \mathrm{e}}$ & 0.3 & $12 \cdot 2^{A, d}$ & 1.0 & $12 \cdot 5^{\mathrm{C}, \mathrm{c}, \mathrm{d}}$ & 0.7 & $13 \cdot 2^{A, C}$ & 0.5 & $15 \cdot 9^{\mathrm{A}, \mathrm{a}}$ & 0.4 & $11 \cdot 7^{\mathrm{B}, \mathrm{d}}$ & 0.1 \\
\hline 18 months & $16 \cdot 3^{A, a}$ & 0.5 & $12 \cdot 7^{\mathrm{A}, \mathrm{C}}$ & 0.2 & $13 \cdot 7^{\mathrm{A}, \mathrm{b}}$ & 0.6 & $14 \cdot 4^{\mathrm{A}, \mathrm{b}}$ & 0.1 & $14 \cdot 0^{\mathrm{A}, \mathrm{b}}$ & 0.3 & $16 \cdot 1^{\mathrm{A}, \mathrm{a}}$ & 1.0 & $13 \cdot 4^{\mathrm{A}, \mathrm{b}, \mathrm{c}}$ & 0.6 \\
\hline
\end{tabular}

a,b,c,d,e,f Mean values within the same row with unlike superscript letters were significantly different $(P<0.05)$.

$A, B, C, D$ Mean values within the same column with unlike superscript letters were significantly different $(P<0 \cdot 05)$. 
DHA $(15.3 \%)$, while the medulla had the least $(11.7 \%)$ among the seven regions examined. Similarly, AA was the highest $(12.8 \%)$ in the cortex while it was the lowest in the medulla $(8.0 \%)$. The difference in 16:0 was also significant among the seven regions, with the cortex having $26.0 \%$ while the medulla had only $17.2 \%$. The medulla had the highest percentage of $18: 1 n-9(22 \cdot 3)$ while the cortex had the lowest percentage of $18: 1 n-9(13 \cdot 13)$.

The distribution pattern of brain fatty acids in adult rats (10 weeks) is shown in Table 4. DHA was the highest in the cortex $(15.9 \%)$ followed by that in the cerebellum (14.2\%), hippocampus $(13.2 \%)$, striatum $(12.5 \%)$, hypothalamus $(12.2 \%)$, midbrain $(11.7 \%)$ and medulla $(10.7 \%)$. AA was the greatest in the hippocampus $(11.4 \%)$ and the least in medulla $(5.6 \%)$. The fatty acid 18:1n-9 was accumulated most in the medulla $(26.5 \%)$ and least in the cortex $(17.8 \%)$.

Distribution pattern was assessed for aged rats (18 months). Regarding DHA, the cerebellum (16.3\%) and cortex (16.1\%) accumulated most while the medulla $(12.7 \%)$ and midbrain $(13.4 \%)$ had the least. In the case of AA, the hippocampus had most $(10.4 \%)$ whereas the medulla contained the least $(5.0 \%)$. Proportionally, the cortex had the greatest percentage of $16: 0$ $(21.8)$ in contrast to the least percentage in the medulla (13.9). In contrast, the medulla accumulated $18: 1 n-9$ the most $(26 \cdot 6 \%)$ whereas in the cortex it was only $19 \cdot 0 \%$.
Ageing-induced change in regional docosahexaenoic acid distribution in brain

Ageing affected DHA distribution most in the cerebellum, where DHA percentage increased with ageing. In contrast, age-induced change in DHA distribution was not so obvious in the other regions. Regarding other fatty acids, ageing led to a decrease in percentages of AA, 22:4n-6, 22:5n-6 and $16: 0$ in all seven regions. The reverse was true for that of $18: 1$, which increased with ageing.

\section{$\alpha$-Linolenic acid deficiency-induced docosahexaenoic acid depletion in brain sub-regions}

Feeding the $n$-3-deficient diet caused depletion of DHA in the brain of F2 female rats (aged 5 weeks). As shown in Table 5, DHA was reduced in various regions by $57 \cdot 1-75.7 \%$. The cortex, hippocampus, cerebellum and striatum appeared to have DHA depleted the most $(73 \cdot 2-75.7 \%)$ followed by the hypothalamus $(71.5 \%)$, midbrain $(64.4 \%)$ and medulla $(57.1 \%)$. Depletion of DHA was mainly compensated by an increase in $22: 5 n-6$ by $7-14$-fold across various regions of rat brain. Feeding the $n-3$ deficient diet led to the medulla accumulating $22: 5 n-6$ the most (14-fold) while the hypothalamus accumulated $22: 5 n-6$ the least (only 8-fold).

Table 5. Alteration in fatty acid composition of brain sub-regions in F2 $n$-3-deficient rats ( $n$-3 Def) compared with that of F2 $n$-3-adequate rats ( $n$-3 Adq) aged 5 weeks ( $n$ 5)

(Mean values and standard deviations for five rats)

\begin{tabular}{|c|c|c|c|c|c|c|c|c|c|c|c|c|c|c|}
\hline & \multicolumn{2}{|c|}{ Cerebellum } & \multicolumn{2}{|c|}{ Medulla } & \multicolumn{2}{|c|}{ Hypothalamus } & \multicolumn{2}{|c|}{ Striatum } & \multicolumn{2}{|c|}{ Hippocampus } & \multicolumn{2}{|c|}{ Cortex } & \multicolumn{2}{|c|}{ Midbrain } \\
\hline & Mean & SD & Mean & $\mathrm{SD}$ & Mean & SD & Mean & SD & Mean & SD & Mean & SD & Mean & SD \\
\hline$n-3$ Adq & $21 \cdot 2$ & 0.8 & $15 \cdot 2$ & 0.3 & $21 \cdot 2$ & 1.4 & $21 \cdot 5$ & 0.4 & $22 \cdot 9$ & 0.3 & $23 \cdot 8$ & 0.3 & $19 \cdot 3$ & 0.9 \\
\hline$n-3$ Def & $21 \cdot 6$ & 0.8 & $15 \cdot 5$ & 0.6 & $22 \cdot 6$ & 0.9 & $22 \cdot 4$ & $1 \cdot 1$ & $24 \cdot 1$ & $1 \cdot 1$ & $22 \cdot 5$ & 0.9 & $19 \cdot 4$ & 0.5 \\
\hline$\%$ Change & $2 \cdot 2$ & 0.1 & 1.5 & 0.1 & $6 \cdot 7$ & 0.3 & $4 \cdot 2$ & 0.2 & $5 \cdot 5$ & 0.3 & $-5 \cdot 2$ & 0.2 & 0.7 & 0.0 \\
\hline \multicolumn{15}{|l|}{$18: 0$} \\
\hline$n-3$ Adq & $20 \cdot 9$ & 0.5 & $20 \cdot 5$ & 0.8 & $22 \cdot 4$ & 0.7 & 23.5 & 1.4 & $22 \cdot 5$ & 0.7 & $22 \cdot 6$ & 0.3 & $22 \cdot 3$ & 0.3 \\
\hline$n-3$ Def & 20.2 & 0.5 & $19 \cdot 8$ & 0.4 & $22 \cdot 7$ & 1.0 & 23.6 & 1.6 & $23 \cdot 2$ & 1.0 & $23 \cdot 2$ & 1.0 & $22 \cdot 0$ & 0.3 \\
\hline$\%$ Change & $-3.4^{b}$ & 0.0 & $-3 \cdot 6^{b}$ & 0.1 & $1 \cdot 0^{\mathrm{a}}$ & 0.0 & $0.8^{a}$ & 0.1 & $3.3^{a}$ & 0.1 & $3.3^{a}$ & 0.1 & $-1 \cdot 5^{\mathrm{b}}$ & 0.0 \\
\hline \multicolumn{15}{|l|}{$18: 1 n-9$} \\
\hline$n-3$ Adq & $19 \cdot 1$ & 0.5 & $24 \cdot 4$ & 0.6 & $19 \cdot 2$ & 0.4 & $17 \cdot 7$ & 0.5 & $17 \cdot 3$ & 0.2 & $16 \cdot 0$ & 0.7 & $20 \cdot 6$ & $1 \cdot 2$ \\
\hline$n-3$ Def & $18 \cdot 1$ & 0.3 & $22 \cdot 5$ & 0.7 & $17 \cdot 7$ & 0.6 & $16 \cdot 5$ & 0.8 & $16 \cdot 1$ & 0.8 & $14 \cdot 6$ & 0.8 & $19 \cdot 3$ & 0.8 \\
\hline$\%$ Change & $-5 \cdot 1$ & 0.1 & $-7 \cdot 7$ & 0.2 & $-8 \cdot 1$ & 0.3 & $-6 \cdot 7$ & 0.3 & -6.9 & 0.3 & -8.4 & 0.5 & -6.4 & 0.3 \\
\hline \multicolumn{15}{|l|}{$18: 2 n-6$} \\
\hline$n-3$ Adq & $1 \cdot 0$ & 0.1 & 0.8 & 0.1 & 0.6 & 0.1 & 0.7 & 0.0 & 0.6 & 0.0 & 0.6 & 0.1 & 0.6 & $0 \cdot 1$ \\
\hline$\%$ Change & $-13 \cdot 5$ & 1.7 & -8.6 & $1 \cdot 2$ & $-8 \cdot 2$ & 1.0 & $-25 \cdot 8$ & $17 \cdot 4$ & $-32 \cdot 7$ & $17 \cdot 4$ & 9.4 & 0.8 & $-15 \cdot 9$ & 3.6 \\
\hline \multicolumn{15}{|l|}{$20: 4 n-6$} \\
\hline$n-3$ Adq & $7 \cdot 4$ & 0.2 & 6.5 & 0.3 & $9 \cdot 2$ & 0.5 & $10 \cdot 3$ & 0.3 & $11 \cdot 1$ & 0.3 & $10 \cdot 3$ & $1 \cdot 1$ & 8.5 & 0.8 \\
\hline$n-3$ Def & $7 \cdot 9$ & 0.1 & $7 \cdot 0$ & 0.3 & $9 \cdot 0$ & 0.2 & $10 \cdot 4$ & 0.7 & $10 \cdot 4$ & 0.7 & $10 \cdot 7$ & 0.5 & 8.4 & 0.8 \\
\hline$\%$ Change & $7 \cdot 5$ & 0.1 & $7 \cdot 2$ & 0.3 & -1.8 & 0.1 & 0.2 & 0.0 & -3.4 & 0.2 & -3.2 & 0.2 & -0.6 & 0.1 \\
\hline \multicolumn{15}{|l|}{$22: 4 n-6$} \\
\hline$n-3 \mathrm{Adq}$ & $2 \cdot 5$ & 0.1 & $3 \cdot 1$ & 0.2 & $4 \cdot 3$ & 0.2 & 3.0 & 0.2 & $3 \cdot 3$ & 0.1 & $3 \cdot 1$ & 0.3 & $3 \cdot 4$ & 0.1 \\
\hline$n-3$ Def & 3.4 & 0.1 & 3.6 & 0.4 & 4.0 & 0.1 & $3 \cdot 1$ & 0.4 & $3 \cdot 2$ & 0.4 & $3 \cdot 3$ & 0.3 & $3 \cdot 7$ & 0.3 \\
\hline$\%$ Change & $35 \cdot 7^{\mathrm{a}}$ & $2 \cdot 0$ & $14 \cdot 9^{\mathrm{b}}$ & 1.5 & $-17 \cdot 9^{c}$ & 0.2 & $6 \cdot 4^{\mathrm{b}, \mathrm{c}}$ & 0.8 & $-2 \cdot 7^{c}$ & 0.3 & $7 \cdot 8^{\mathrm{bc}}$ & 0.7 & $11 \cdot 0^{\mathrm{b}}$ & 0.9 \\
\hline \multicolumn{15}{|l|}{$22: 5 n-6$} \\
\hline$n-3$ Adq & 0.8 & 0.1 & 0.5 & 0.0 & $1 \cdot 1$ & 0.2 & 0.9 & 0.1 & 1.0 & 0.1 & 1.0 & 0.2 & 0.8 & 0.2 \\
\hline$n-3$ Def & $10 \cdot 6$ & 0.8 & $7 \cdot 0$ & 0.4 & $9 \cdot 4$ & 0.9 & $10 \cdot 6$ & $1 \cdot 1$ & $10 \cdot 0$ & 0.3 & $11 \cdot 6$ & 1.0 & $9 \cdot 4$ & 0.5 \\
\hline$\%$ Change & $1256^{a, b}$ & 39 & $1420^{a}$ & 8 & $790^{\circ}$ & 74 & $1041^{b c}$ & 105 & $873^{c}$ & 29 & $1058^{\mathrm{b}}$ & 94 & $1117^{b}$ & 54 \\
\hline \multicolumn{15}{|l|}{$22: 6 n-3$} \\
\hline$n-3$ Adq & $13 \cdot 8$ & 3.7 & $10 \cdot 0$ & 0.4 & $12 \cdot 2$ & 0.6 & $12 \cdot 5$ & 0.7 & $12 \cdot 3$ & 0.7 & $13 \cdot 9$ & 0.5 & $12 \cdot 2$ & 0.6 \\
\hline$n-3$ Def & 3.7 & 0.6 & $4 \cdot 3$ & 0.2 & 3.5 & 0.3 & 3.4 & 0.4 & $3 \cdot 1$ & 0.4 & 3.4 & 0.6 & 4.4 & 0.2 \\
\hline$\%$ Change & $-73.4^{c}$ & $12 \cdot 6$ & $-57 \cdot 1^{a}$ & 2.5 & $-71 \cdot 5^{\mathrm{c}}$ & $6 \cdot 8$ & $-73 \cdot 2^{c}$ & 8.7 & $-74 \cdot 4^{c}$ & 9.5 & $-75 \cdot 7^{\mathrm{c}}$ & 12.9 & $-64 \cdot 4^{\mathrm{b}}$ & 3.0 \\
\hline
\end{tabular}

a,b,c,d Mean values within the same row with unlike superscript letters were significantly different $(P<0.05)$. 


\section{Reversibility of docosahexaenoic acid deficiency}

DHA depletion in the brain was reversible after the $n$-3 Def rats were switched to the $n-3$ Adq diet (Fig. 1). There was about a $50 \%$ recovery of DHA in seven regions within the first 3 weeks. After 12 weeks of diet reversal, DHA recovery was almost complete in all regions except for the medulla where DHA percentage was only 62 of that in the $n-3$ Adq rats. Fatty acid 22:5n-6 was an indicator of DHA deficiency. It rose by $7-14$-fold across various regions in the $n-3$ Def rats compared with that in the $n-3$ Adq rats (Table 5). Fatty acid 22:5n-6 in the $n-3$ Def group gradually decreased and approached that of the $n$-3 Adq group after 12 weeks of the diet reversal, but statistically it was still higher in the $n-3$ Def group than that in the $n-3$ Adq control (Fig. 2).

\section{Discussion}

The present study examined fatty acid composition across various regions in the rat brain with its focus entirely on DHA and AA.
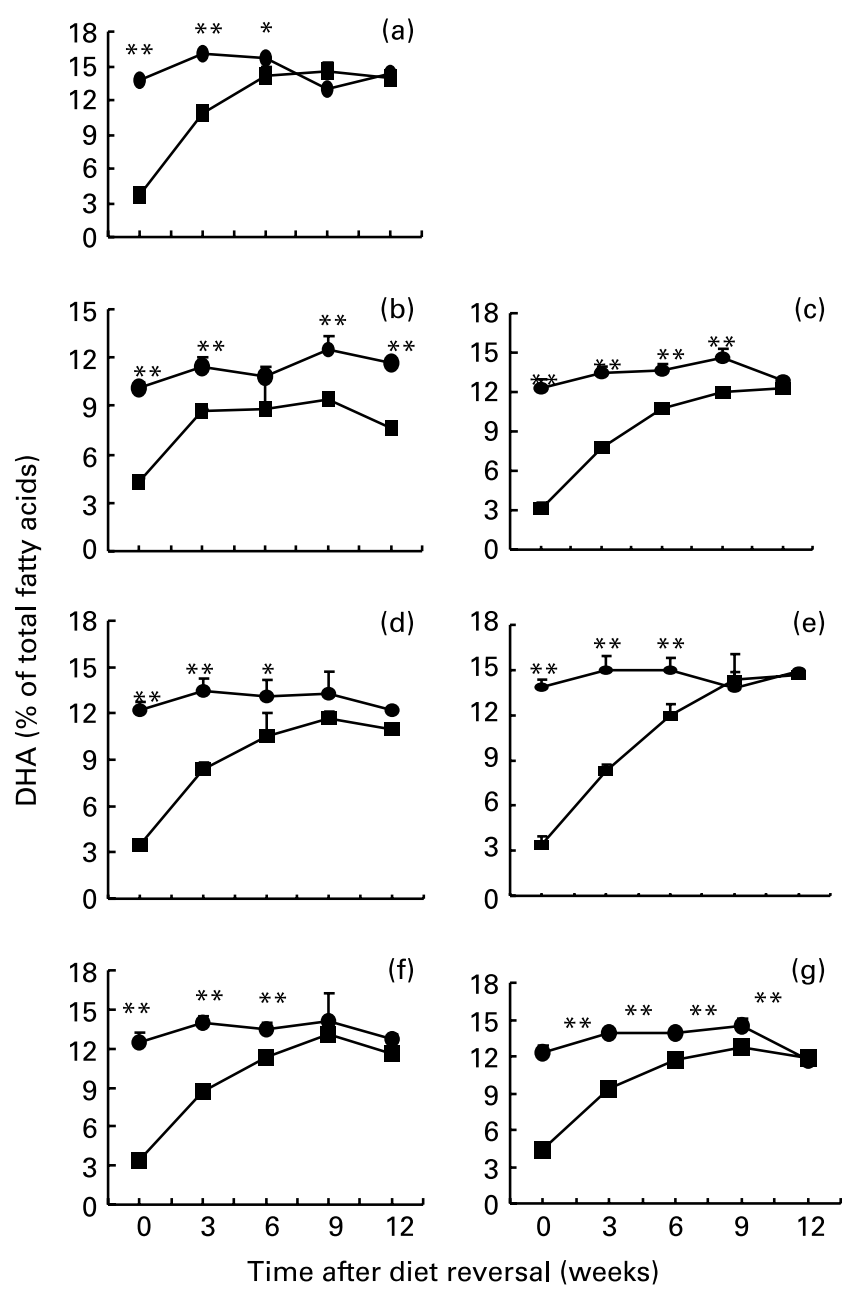

Fig. 1. Regional changes in DHA (\% of total fatty acids) in the cerebellum (a), medulla (b), hippocampus (c), hypothalamus (d), cortex (e), striatum (f) and midbrain $(\mathrm{g})$ of $\mathrm{F} 2$ female rats after switching from an $n$-3-deficient diet ( $n$-3 Def; $\mathbf{\square})$ to an $n$-3-adequate diet ( $n$-3 Adq; $\bullet$ ). At each time point, the values are means for five rats, with standard deviations represented by vertical bars. Mean value was significantly different from that of the $n-3$ Def rats: ${ }^{\star} P<0.05 ;{ }^{* \star} P<0.01$.
The results clearly demonstrated that fatty acid composition varied with various regions and it was evident that DHA and AA did not distribute evenly in the brain. The present study found that in weaning and adult rats the region with the highest DHA percentage was the cortex whereas the medulla had the lowest DHA percentage. This observation was similar to the report by Carrie et al. (2000), who examined the fatty acid composition of PL present in various regions in adult mice. The present study was carried out to investigate further the age-related changes in DHA levels of various brain regions, demonstrating that in aged rats (18 months), the cerebellum and cortex were the regions that equally had the highest DHA percentages compared with other regions in the brain (Table 4). Regarding AA, the hippocampus was the region that always had the highest percentage whereas the medulla was the region with the lowest AA percentage except for the neonatal rats (aged $3 \mathrm{~d}$ ), whose difference in AA across various regions was not so obvious and apparent, although statistically the hippocampus and cortex had an AA percentage higher than the cerebellum, hypothalamus, striatum
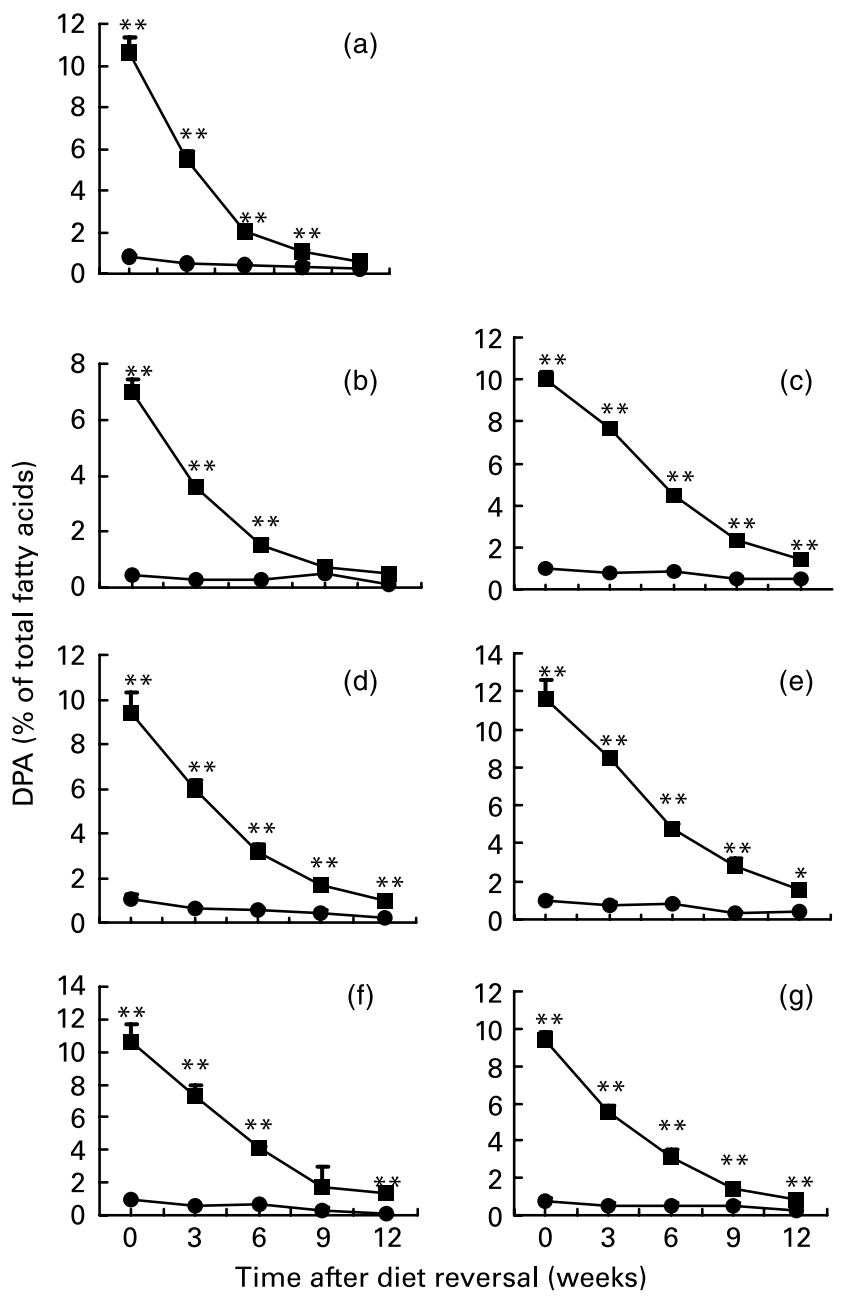

Fig. 2. Regional changes in docosapentaenoic acid (DPA, \% of total fatty acids) in the cerebellum (a), medulla (b), hippocampus (c), hypothalamus (d), cortex (e), striatum (f) and midbrain (g) of F2 female rats after switching from an $n$-3-deficient diet ( $n$-3 Def; $\square$ ) to an $n$-3-adequate diet ( $n$-3 Adq; $\bullet$ ). At each time point, the values are means for five rats, with standard deviations represented by vertical bars. Mean value was significantly different from that of the $n-3$ Def rats: ${ }^{\star} P<0.05 ;{ }^{* *} P<0.01$. 
and midbrain. The present result agreed with that reported by Carrie et al. (2000) on 4-month-old mice, but it had no comparison with the data presented by Marteinsdottir et al. (1998) and Favreliere et al. (1998), who measured fatty acid composition in other regions rather than the medulla.

The difference in distribution of DHA and AA across various regions was apparently greater when the neonatal rats were compared with the weaning and adult rats. In the neonatal rats (aged $3 \mathrm{~d}$ ), the difference in DHA content was less than $15 \cdot 1 \%$ between the striatum with the highest percentage (12.6) and the hypothalamus that had the lowest percentage (10.7). The weaning rats had a difference of $23.5 \%$ when DHA content in the cortex (15.3\%) was compared with that in the medulla $(11.7 \%)$. It was true that the difference in DHA content was even greater $(32.7 \%)$ between the cortex $(15.9 \%)$ and medulla $(10.7 \%)$. This difference could be greater $(>59.3 \%)$ in mice as reported by Carrie et al. (2000), who found that in 4-month-old mice, the medulla had a DHA content of $9.0 \%$ while front cortex had a DHA content of $22.1 \%$. It was more evident if the difference in AA content in a region that had the highest percentage was compared with that with the lowest percentage. The neonatal rats (day 3) had a difference less than $4.7 \%$ (hippocampus, $12.9 \%$; striatum, $12.3 \%$ ). In contrast, the weaning rats (day 21) had a difference increased to $37.5 \%$ (cortex, $12.84 \%$; medulla, $8.02 \%$ ) while the adult rats (10 weeks) had a difference greater than $50.9 \%$ (hippocampus, $11.4 \%$; medulla, 5.6\%). Similarly, Carrie et al. (2000) observed that, in mice, the medulla had the lowest AA percentage (4.7) while hippocampus had the highest AA percentage $(10 \cdot 5)$.

The present study showed that DHA was not proportionally depleted in various regions of brain in response to an $n$ - 3 deficient diet (Table 5). The cortex, hippocampus, striatum, cerebellum and hypothalamus had DHA depleted by more than $71 \%$ followed by the midbrain, which had $64 \%$ depletion. In contrast, the medulla appeared to be a region that was relatively resistant to depletion, having DHA depleted only by $57 \%$ (Table 5). The present study also showed that $n-3$ deficiency was characterised by not only a decrease in brain DHA percentage but also an increase in 22:5n-6 (Table 5). This observation is in agreement with a report on rats by Moriguchi et al. (2001), mice by Carrie et al. (2000) and monkeys by Connor et al. (1990). Compared with the $n$ - 3 adequate diet, $22: 5 n-6$ increased by $7-14$-fold in various brain regions of rats maintained on the $n$-3-deficient diet, suggesting that the depletion of DHA was compensated by an accumulation of $22: 5 n-6$ in the brain. In this regard, the medulla had 22:5n-6 accumulated most although it had DHA lost least (Table 5). In contrast, the hypothalamus and hippocampus were the two regions that gained $22: 5 n-6$ least.

The present study is the first to examine the regional difference in DHA recovery in rat brain. The major finding was that the medulla exhibited a much slower time course for DHA recovery compared with the other regions (Fig. 1). The present study is in agreement with that by Moriguchi et al. (2001), who only examined the rat brain as a whole and found that full recovery of DHA in F2 n-3-deficient rats was not possible until 8 weeks after switching from an $n$-3-deficient diet to an $n$-3 adequate diet. The similar DHA reversible rate in retina was also observed, but DHA recovery in the liver and serum was complete within 2 weeks after the diet reversal (Moriguchi et al. 2001). Connor et al. (1990) studied reversibility of $n$-3 fatty acid deficiency in the brain of rhesus monkeys and found that DHA level in the cerebral cortex could be up regulated by switching to feeding a fish oil diet.

Each brain region is associated with its specific functions (Bennett, 1977). For example, the cerebellum is the site related to the control of balance in body movement, and the storage of conditional learning. The hypothalamus has been implicated in the mediation of a number of behaviours including sleep, waking, emotion, reproductive activity, hunger and thirst (Bennett, 1977). The cerebral cortex and hippocampus are the sites that are involved in information storage and control of memory, intelligence, thoughts and other higher mental functions. It has been shown that the level of brain DHA falling is associated with brain functional changes. In human infants, the addition of DHA or DHA and AA in combination into infant formula improves performance in visual acuity, visual recognition memory, learning ability, Bayley mental developmental index, and developmental quotient scores (Uauy et al. 1990; Carlson et al. 1993; Agostoni et al. 1995; Carlson \& Werkman, 1996; Willatts et al. 1998; Birch et al. 2000; Innis, 2004). When maintained on an $n$-3-deficient diet, rats or mice had a poorer performance in a Y-maze (Lamptey \& Walker, 1976), shock avoidance (Bourre et al. 1989) and spatial learning (Moriguchi et al. 2000). When the rats or mice were re-fed with the $n-3$ fatty acid-supplemented diet, the DHA level was partially recovered and so were spatial learning (Moriguchi \& Salem, 2003) and the performance behaviour in a maze (Lim \& Suzuki, 2001). The present results demonstrated that the cortex and hippocampus were the two sites where DHA was depleted most (Table 5). This correlates well with the observation that the rats showed poorer performance in a maze, shock avoidance and spatial learning when fed the $n$-3-deficient diet.

In summary, the present results demonstrated that DHA and AA in the brain were not proportionally distributed in various regions. The distribution of DHA and AA was age-dependent and region-specific. Most important was that DHA in the brain was not proportionally depleted in response to an LNA-deficient diet. Most interesting was that complete recovery of DHA was possible in all sub-regions except for the medulla, where DHA recovery reached only $62 \%$ after 12 weeks of diet reversal.

\section{References}

Agostoni C, Trojan S, Bellu R, Riva D \& Giovannini M (1995) Neurodevelopment quotient of healthy term infants at 4 months and feeding practice: the role of long-chain polyunsaturated fatty acids. Pediatr Res 38, 262-266.

Auestad N, Montalto MB, Hall RT, Fitzgerald KM, Wheeler RE, Connor WE, Neuringer M, Connor SL, Taylor JA \& Hartmann EE (1997) Visual acuity, erythrocyte fatty acid composition, and growth in term infants fed formulas with long chain polyunsaturated fatty acids for one year. Ross Pediatric Lipid Study. Pediatr Res 41, 1-10.

Bennett TL (1977) The anatomy of the brain. In Brain and Behavior, pp. 24-50. Monterey, CA: Brooks/Cole Publishing Company.

Birch EE, Garfield S, Hoffman DR, Uauy R \& Birch DG (2000) A randomized controlled trial of early dietary supply of long-chain polyunsaturated fatty acids and mental development in term infants. Dev Med Child Neurol 42, 174-181.

Bourre JM, Francois M, Youyou A, Dummont D, Piciotti M, Pascal G \& Durand G (1989) The effects of dietary alpha-linolenic acid on the composition of nerve membrane, enzymatic activity, amplitude of electrophysiological parameters, resistance to poison and performance of learning tasks in rats. $J$ Nutr 119, 1880-1892. 
Carlson SE \& Werkman SH (1996) A randomized trial of visual attention of perterm infants fed docosahexaenoic acid until two months. Lipids 31, 85-90.

Carlson SE, Werkman SH, Rhodes PG \& Tolley EA (1993) Visual-acuity development in healthy preterm infants; effect of marine-oil supplementation. Am J Clin Nutr 58, 35-42.

Carrie I, Clement M, de Javel D, Fances H \& Bourre JM (2000) Specific phospholipid fatty acid composition of brain regions in mice: effect of $n-3$ polyunsturated fatty acid deficiency and phospholipid supplementation. J Lipid Res 41, 465-472.

Connor WE, Neuringer M \& Lin DS (1990) Dietary effect on brain fatty acid composition: the reversibility of $n-3$ fatty acid deficiency and turnover of docosahexaneoic acid in the brain, erythrocytes, and plasma of rhesus monkeys. J Lipid Res 31, 237-247.

Enslen M, Milon H \& Malnoe A (1991) Effect of low intake of $n-3$ fatty acids during development on brain phospholipids fatty acid composition and exploratory behavior in rats. Lipids 26, 203-208.

Favreliere S, Barruer L, Durand G, Chalon S \& Tallibeay C (1998) Chronic dietary $n-3$ deficiency affects the fatty acid composition of plasmenylethanolamine and phosphatidylethanolamine differently in rat frontal cortex, striatum and cerebellum. Lipids 33, 401-407.

Glowinski J \& Iversen LL (1966) Regional studies of catecholamines in the rat brain. I. The disposition of $\left[{ }^{3} \mathrm{H}\right]$ norepinephrine, $\left[{ }^{3} \mathrm{H}\right]$ dopamine and $\left[{ }^{3} \mathrm{H}\right]$ DOPA in various regions of the brain. J Neurochem 13, 655-669.

Greiner RS, Moriguchi T, Hutton A, Slotnick BM \& Salem N (1999) Rats with low level of brain docosahexaenoic acid show impaired performance in olfactory-based and spatial learning tasks. Lipids 34, S239-S243.

Innis SM (2004) Polyunsaturated fatty acids in human milk: an essential role in infant development. Ad Exp Med Biol 554, 27-43.

Innis SM, Nelson CM, Rioux MF \& King DJ (1994) Development of visual acuity in relation to plasma and erythrocyte omega- 6 and omega-3 fatty acids in healthy term gestation infants. Am J Clin Nutr 60, 347-352.

Jorgensen MH, Holmer G, Lund P, Hernell O \& Michaelsen KF (1998) Effect of formula supplemented with docosahexaenoic acid and gamma-linolenic acid on fatty acid status and visual acuity in term infants. J Pediatr Gastroenterol Nutr 26, 412-421.

Lamptey MS \& Walker BL (1976) A possible essential role for dietary linolenic acid in the development of the young rat. J Nutr 106, 86-93.

Lim SY \& Suzuki H (2001) Changes in maze behavior of mice occur after sufficient accumulation of docosahexaenoic acid in brain. J Nutr 131, 319-324.

Lucas A, Morley R, Stephenson T \& Elias-Jones A (2002) Long-chain polyunsaturated fatty acids and infant formula [Letter]. Lancet 360, 1178.
Lucas A, Stafford M, Morley R, Abbott R, Stephenson T, MacFadyen U \& Elias-Jones A (1999) Efficacy and safety of long-chain polyunsaturated fatty acid supplementation of infant-formula milk: a randomized trial. Lancet 354, 1948-1954.

Makrides M, Neumann MA, Simmer K \& Gibson R (1995) Are longchain polyunsaturated fatty acids essential nutrients in infancy? Lancet 345, 1463-1468.

Marteinsdottir I, Horrobin DF, Stenfors C, Theodorsson E \& Mathe AA (1998) Changes in dietary fatty acids alter phospholipid fatty acid composition in selected regions of rat brain. Prog Neuropsychopharmacol Biol Psychiatry 22, 1007-1021.

Moriguchi T, Greiner RS \& Salem N (2000) Behavioral deficits associated with dietary induction of decreased brain docosahexaenoic acid concentration. J Neurochem 75, 2563-2573.

Moriguchi T, Loewke J, Garrison M, Catalan JN \& Salem N (2001) Reversal of docosahexaenoic acid deficiency in the rat brain, retina, liver and serum. J Lipid Res 42, 419-427.

Moriguchi T \& Salem N (2003) Recovery of brain docosahexaneoate leads to recovery of spatial task performance. J Neurochem 87, 297-309.

Neuringer MW, Connor E, Lin DS, Barstad L \& Luck S (1986) Biochemical and functional effects of prenatal and postnatal omega 3 fatty acid deficiency on retina and brain in rhesus monkeys. Proc Natl Acad Sci U S A 83, 4021-4025.

Pawlosky RJ, Denkins Y, Ward G \& Salem N (1997) Retinal and brain accretion of long-chain polyunsaturated fatty acids in developing felines: the effect of corn oil-based maternal diets. Am J Clin Nutr 65, 465-472.

Sastry P (1985) Lipid of nervous tissue:composition and metabolism. Prog Lipid Res 24, 69-176.

Uauy RD, Birch DG, Birch EE, Tyson JE \& Hoffman DR (1990) Effect of dietary omega-3 fatty acids on retinal function of very low birth weight neonates. Pediatr Res 28, 485-492.

Wainwright PE, Huang YS, Coscina DV, Levesque S \& McCutcheon D (1994) Brain and behavioral effect of dietary $n-3$ deficiency in mice: a three generation study. Dev Psychobiol 27, 467-487.

Wainwright PE, Xing HC, Mutsaers L, McCutcheon D \& Kyle D (1997) Arachidonic acid offsets the effects on mouse brain and behavior of a diet with a low $(n-6 / n-3)$ ratio and very high levels of docosahexaenoic acid. J Nutr 127, 184-193.

Wheeler TG, Benolken RM \& Anderson RE (1975) Visual membrane: specificity of fatty acid precursors for the electrical response to illumination. Science 188, 1312-1314.

Willatts P, Forsyth JS, Dimodugno MK, Varma S \& Colvin M (1998) Effect of long-chain polyunsaturated fatty acids in infant formula on problem solving at 10 months of age. Lancet 352, 688-691. 